\title{
Analysing Performances of Dynamic Routing Protocols on Various Network Topologies
}

\author{
Irem Dogan and Erkan Bostanci
}

\begin{abstract}
Dynamic routing protocols are generally used in large-scale networks and routers, which are used to route data packages from own network to remote networks, can be managed easily by network administrator because dynamic routing protocols learn all of the network information dynamically. Performance of a dynamic routing protocol can change with different network topologies. Aim of dynamic routing protocols, types of dynamic routing protocols, analyses of dynamic routing protocols on different network topologies were clarified. Operations of dynamic routing protocols were analysed on different network topologies. "Which dynamic routing protocol should be used ring, star and mesh topology for high performance? Why?" questions were answered with this analysis.
\end{abstract}

Index Terms-Best paths, dynamic routing protocols, performance, routing table.

\section{INTRODUCTION}

End user devices, switch, hub, router etc. are network devices in basic network architecture. These devices work on different layers with different duties. Routers are important devices for a network. These devices are used to route network traffic to other networks. Routing is a process which is performed by routers for routing data packages from a network to others.

The literature presents a number of static and dynamic routing protocols that are used to control the flow of the packages through the network. For many companies at various scales, it is very crucial to choose and construct a network topology and select the most appropriate routing algorithm that fits best to their current topologies, if they wish to have a more efficient network infrastructure.

This paper analyses the performances of different routing protocols in a variety of network topologies. The rest of the paper is structured as follows. Section II presents various routing protocols used in the analysis followed by Section III where the tested network topologies are presented. The analysis criteria are described and the results are presented in Section IV. Finally, the paper is concluded in Section V.

\section{ROUTING PROCESS AND DYNAMIC Routing PROTOCOLS}

\section{A. Overview of Routing Process}

Routing process is performed while destination IP address

Manuscript received January 25, 2016; revised June 6, 2016.

The authors are with SAAT Lab, Computer Engineering Department, Ankara University, Turkey (e-mail: irem.dogan03@hotmail.com, ebostanci@ankara.edu.tr) of a data package belongs a remote network. For performing the routing process, routers use their own routing tables. A routing table contains remote networks' IP addresses and best paths which belong to these remote networks. IP addresses are layer 3 addresses so, a package, which is received by router, is opened in layer 3. Source and destination IP addresses are obtained by using the information contained in this package by router. The information of remote network, which contains destination IP address or this address information, can be learnt by using routing table of the router. Again, the best path is determined by using routing table. Router routes the packages over the interface which is determined by using this information.

\section{B. Static Routing and Dynamic Routing}

There are two types routing configurations that can be run on routers. These configurations are applied by network administrators statically or dynamically. In static routing process, network administrator has to configure routers manually to teach information of remote networks. Static routing is generally used on small-scale network architectures. In large-scale network architecture, static routing is a complex process so, it is not preferred on this networks by network administrators. Performing a dynamic routing configurations by using a set of protocols is called Dynamic Routing Process.

Network administrators perform different protocol configurations on routers. These protocols allow routers to learn about remote networks. Also routers communicate with each other to understand the network topology which they belong to. Thus, routers create their routing tables by sharing information about remote networks with each other. Dynamic routing is generally used on complex or large-scale networks. In a network which is configured by static routing, routers must be re-configured by the networks administrators when there is a change inside of the network or in one of the remote networks. In a network which is configured by dynamic routing, routers update their routing tables dynamically, when there is a change inside of the network or in one of the remote networks. Thus, there is no need to make any re-configurations by network administrators. Thanks to dynamic routing, routing process can be managed easily on complex and large-scale networks.

\section{Overview of Dynamic Routing Protocols}

There are also two types of dynamic routing protocols; Interior Gateway Protocols and Exterior Gateway Protocols.

Interior Gateway protocols can be divided into 2 categories; Distance-Vector Protocols and Link-State Protocols.

Distance-Vector Protocols use hop counts as the metric to determine best paths which are used for routing process. 
Links-State Protocols choose the best path with respect to qualities and availabilities of links on the routers interfaces.

Exterior Gateway Protocols can be examined under the Path-Vector Protocols category. Path-Vector Protocols contain some characteristics of link-state and Distance-Vector Protocols. They are used between Internet Service Providers or Autonomous Systems. Distance-Vector Protocols send update with each other periodically and these updates are broadcast. For this reason, links become up and down periodically and this situation causes high overhead on links.

Link-State Protocols send update when there is a change on the topology. These updates are multicast. This situation causes an overhead on links. This overhead is less than the overhead of Distance-Vector Protocols. This overhead value is not low enough for high performance between ASs and ISPs. Therefore, Path-Vector Protocols were created for using between ASs and IPSs.

Dynamic Routing Protocols used in analysis;

RIPv1 (Routing Information Protocol version 1): This is an Interior-Gateway Protocol and it belongs to Distance-Vector Protocol category. RIPv1 can be used in basic small-scale networks. It is the first Dynamic Routing Protocol [1].

RIPv2 (Routing Information Protocol version 2): This is an Interior-Gateway Protocol and it belongs to Distance-Vector Protocol category. RIPv2 was created because the performance of RIPv1 was not enough. RIPv2 is called Hybrid Routing Protocol because it also contains some characteristics of Link-State Protocols. RIPv2 can be used in small-scale network [1].

EIGRP (Enhanced Interior Gateway Routing Protocol): This is an Interior-Gateway Protocol and it belongs to Distance-Vector Protocol category. It is appropriate to use in large-scale networks [2].

OSPF (Open Shortest Path First): This is an Interior-Gateway Protocol and it belongs to Link-State Protocol category. It is appropriate to use in large-scale networks [3].

BGP (Border Gateway Protocol): This is an Exterior-Gateway Protocol and it belongs to Path-Vector Protocol category. It generally used between Internet Service Providers or Autonomous Systems.

\section{DIFFERENT TYPES OF TOPOLOGIES USED IN ANALYSIS}

In analysis, different types of topologies were used and they were simplified to ease analysis process. These topologies are given in the following.

\section{A. Ring Topology}

The Ring Topology used in analysis:

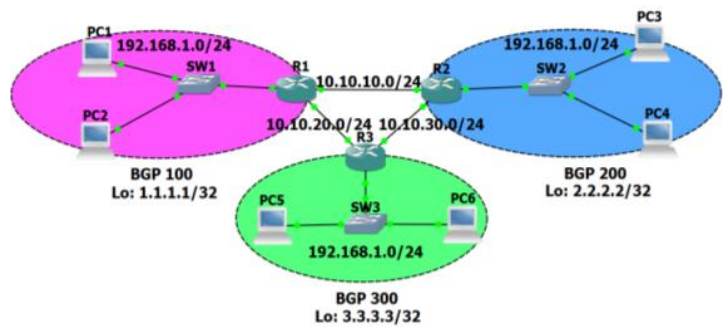

Fig. 1. Ring topology with BGP configuration used in analysis.
As shown in Fig. 1, all of the routers on the network are connected to each other as a ring. If there is a fault on the network, all of the topology is affected this situation.

\section{B. Star Topology}

The Star Topology used in analysis:

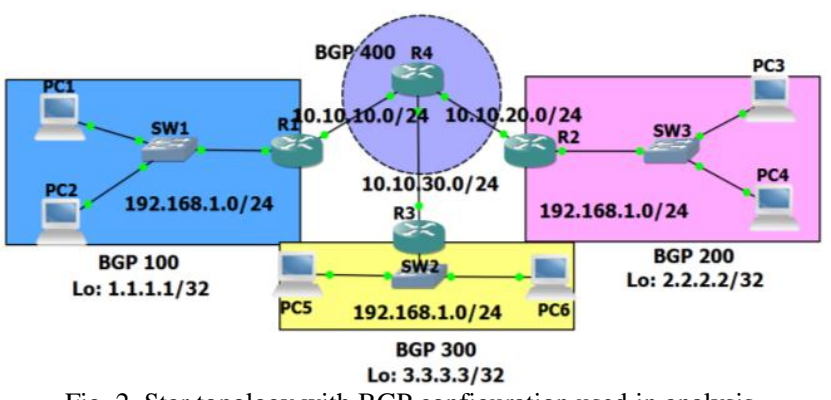

Fig. 2. Star topology with BGP configuration used in analysis.

As shown in Fig. 2, all of the routers on the network are connected to a central router as s star. Thus, if there is a fault on the network, all of the topology is not affected this situation because of using central device.

\section{Mesh Topology}

The Mesh Topology used in analysis:

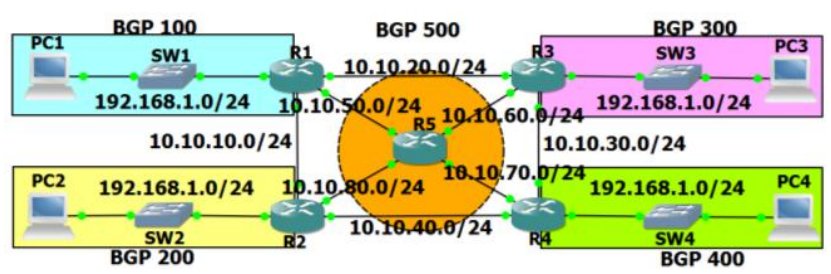

Fig. 3. Mesh topology with BGP configuration used in analysis.

Most of the routers on the network are directly connected each other. As shown in Fig. 3, if all routers are directly connected each other, the topology is called "Full Mesh Topology". This topology provides redundancy between routers, so if there is a fault, all of the network is not affected this situation.

\section{ANALYSIS FRAMEWORK}

RIPv1, RIPv2, EIGRP, OSPF and BGP were used on different types of topologies, and the graphics were created with respect to these protocol configurations on the topologies. Metrics used in analysis can be listed as:

Convergence Time: This is the time takes that the routers to create and update their routing tables with communicating each other by using dynamic routing protocols, in order to learn the information of the network topology. It is identified as second (s).

Overhead: This is the additional load on the link that occurs with using dynamic routing protocols over the routers. It is identified with percentage.

Memory Allocation: This is the memory capacity of a router which is used by dynamic routing protocols and routing process. It is identified with bytes.

Average Internal Round-Trip Time: This is the time takes that a router sends a package on the internal network. We use the ping process for testing. Average Internal Round-Trip 
Time is identified with milliseconds (ms).

Average External Round-Trip Time: This is the time takes that a router sends a package to the remote network. We use the ping process for testing. Average External Round-Trip Time is identified with milliseconds (ms).

\section{A. Analysis of Dynamic Routing Protocols on Ring Topology}

The results of analysis was obtained by using the same ring topology in every experiment but using different dynamic routing protocol in each time. During the analysis, the configurations of RIPv1, RIPv2, EIGRP, OSPF and BGP was done and they were compared with each other.

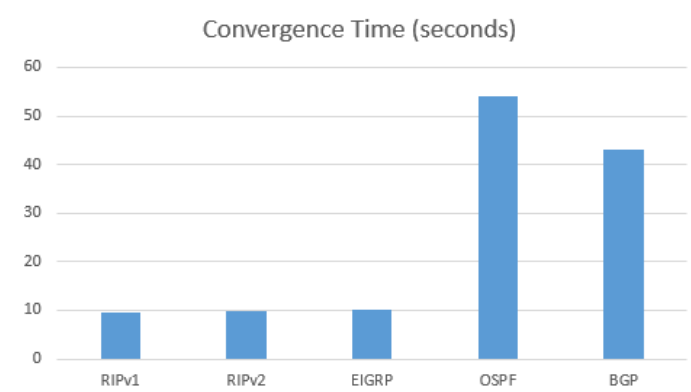

Fig. 4. Convergence time graphic of dynamic routing protocols on ring topology.

As depicted in Fig. 4, considering the interior gateway protocols, the convergence times of RIPv1, RIPv2 and EIGRP are lower when compared to OSPF [4]. The reason why OSPF is higher is that, RIP and EIGRP protocols are distance vector protocols and they periodically receive updates for network's information. Consequently, the router that runs RIP and EIGRP protocols, can detect the changes in network and change their routing tables quicker when compared to OSPF, which is a link state protocol. The convergence time of BGP, which is an exterior gateway protocol, is also high. The reason for this is that BGP was designed to be used in autonomous systems and it runs more complex algorithms, hence, gathering network information is slow.

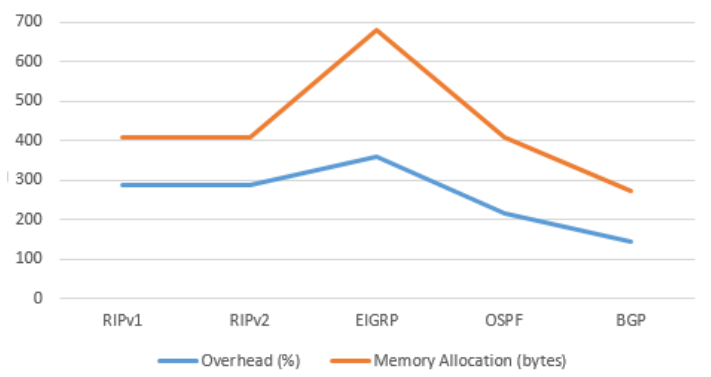

Fig. 5. Overhead and memory allocation graphic of dynamic routing protocols on ring topology.

As given in Fig. 5, considering the overhead, EIGRP has the highest overhead in interior gateway protocols, because it is a distance vector protocol. RIP is also a distance vector protocol, but its working mechanism is simpler than EIGRP, therefore its overhead is lower. The routers that run EIGRP, periodically send update packages between one another. This packages occupy the link and cause the links to be up and down periodically. Therefore, during this periodic updates and the changes in link states, leads to a high overhead. BGP protocol has the lowest overhead value since it is an exterior gateway protocol. BGP's overhead must be low because it is used between autonomous systems. Consequently, the internet connection's continuity could be provided when BGP works with best performance and low overhead. Again as illustrated in Fig. 5, EIGRP is the most memory using protocol in interior gateway protocols. The reason of this memory usage is that EIGRP periodically gets updates from neighbour routers. On the other hand, averagely the same amount of memory usage is observed in the other interior gateway protocols. BGP, which is an exterior gateway protocol, has the lowest memory usage value and this also is a reason why it has a high performance.

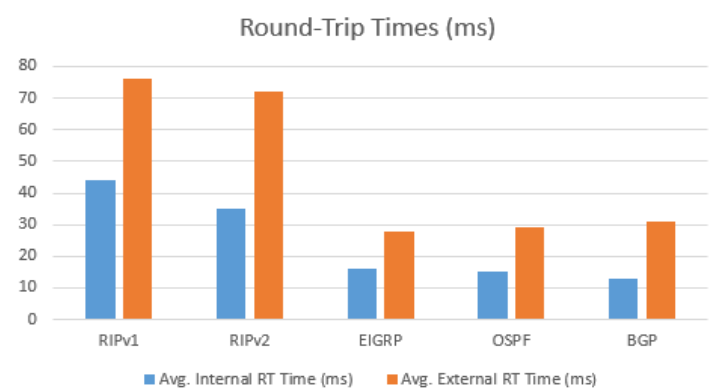

Fig. 6. Average round-trip times graphic of dynamic routing protocols on ring topology.

As shown in Fig. 6, considering the internal protocols, OSPF and EIGRP protocols have approximately the same Average Internal Round-Trip Time values. Average Internal Round-Trip Time values of OSPF and EIGRP is considerably low when compared to RIPv1 and RIPv2. Therefore routers which runs OSPF and EIGRP protocols on them, makes faster and shorter routings. The incoming/outgoing packages to/from this routers reach their destinations faster when compared to other internal gateway protocol using routers. BGP, which is an external gateway protocol, has the lowest Average Internal Round-Trip Time value. This leads a very fast data communication between BGP running routers. Therefore, continuity of internet connection can be provided. Again as shown in Fig. 6, RIPv1 and RIPv2 have the highest Average External Round-Trip Time values between interior gateway protocols. RIP protocols use a simple mechanism and this causes a latency in learning remote network's information and this leads a delay when sending packages to remote networks. OSPF and EIGRP, which are the other interior gateway protocols, show similar Average External Round-Trip Time values. Routers, which runs these protocols, can learn distant networks fast, consequently they can send a package to a distant network faster than routers which run RIP protocols. BGP, which is an exterior gateway protocol, has considerably low round-trip values. This situation leads a fast package transfer and fast network learning between autonomous systems. In the figure below, performances of interior gateway protocols in ring topology is shown in quantile.

As shown in Fig. 7, considering the values above, OSPF has the highest performance between Interior Gateway Dynamic Routing Protocols in ring topology. Therefore, OSPF can be preferred in ring topology structured networks. 
BGP works with higher performance when compared to OSPF, but BGP is an exterior gateway protocol. Accordingly, the use of BGP is convenient in combination of autonomous system networks, not in internal networks.

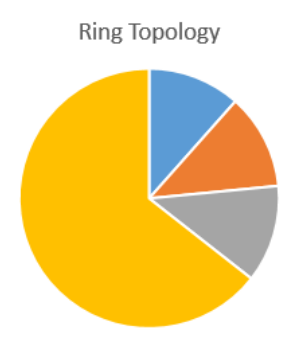

- RIPV1 | RIPV2 | EIGRP $=$ OSP

Fig. 7. Analysis of interior gateway protocols on ring topology.

\section{B. Analysis of Dynamic Routing Protocols on Star Topology}

The results of analysis was obtained by using the same star topology in every experiment but different dynamic routing protocols in each time. During the analysis, the configurations of RIPv1, RIPv2, EIGRP, OSPF and BGP were done and they were compared with each other.

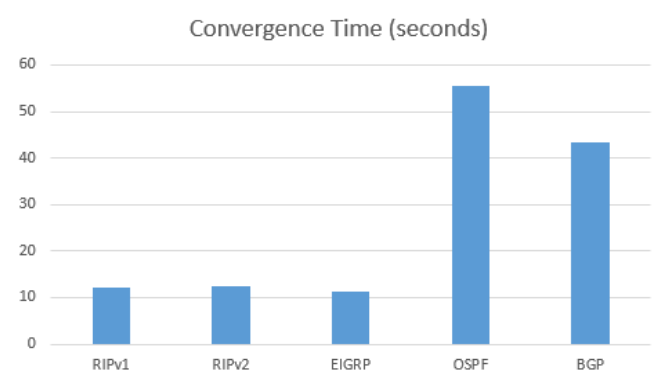

Fig. 8. Convergence time graphic of dynamic routing protocols on star topology.

As shown in Fig. 8, considering the Convergence Time of Dynamic Routing Protocols on Star Topology, EIGRP has the lowest convergence time. Routers, which run EIGRP, create and update their routing tables faster when compared to other routers running other interior gateway protocols. Consequently, EIGRP has more advantages in star topology networks if convergence time is considered. OSPF has the highest convergence time value between interior gateway protocols. Routers, which run OSPF, lost more time in star topology networks than routers, which run other protocols, when creating their routing tables. Consequently, using OSPF protocol in star topology networks would be a disadvantage in terms of convergence time. The answer of why BGP, which is an exterior gateway protocol, has a high convergence value is that, it runs a complex algorithm to discover networks.

As depicted in Fig. 9, considering the overhead, EIGRP has the highest overhead value between interior gateway protocols [5]. Same situations explained in Ring Topology Overhead occur in star topology. EIGRP is a distance vector protocol. This makes EIGRP to be a high overhead protocol because updates will be sent periodically from the network. In star topology, OSPF, which is a dynamic routing protocol, causes the lowest overhead value. Router's cause of overhead in links, which run OSPF, would be quite low in star topology. The reason of this is that, OSPF is a link state protocol and it takes information of the network and updates from the core device of the network, which knows whole network. Therefore, it works quite fast. BGP, which is an exterior protocol, has also low value of overhead because BGP works between autonomous systems so it is designed to provide a continuous and high quality internet connection and work with low overhead values. Again as shown in Fig. 9, between interior gateway protocols, EIGRP is the most memory using protocol [6]. Similar conditions in Ring Topology also come up in Star Topology. Memory usage value of EIGRP would be also the highest in Star Topology when compared to other protocols, because it would again get updates periodically from neighbour routers. BGP, which is an exterior gateway protocol, has the lowest memory usage value because BGP works between autonomous systems, so it's design aim is using minimum memory to provide maximum performance.

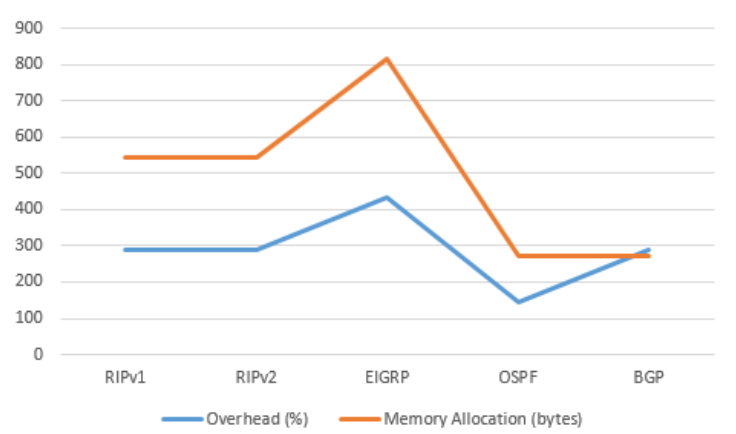

Fig. 9. Overhead and memory allocation graphic of dynamic routing protocols on star topology.

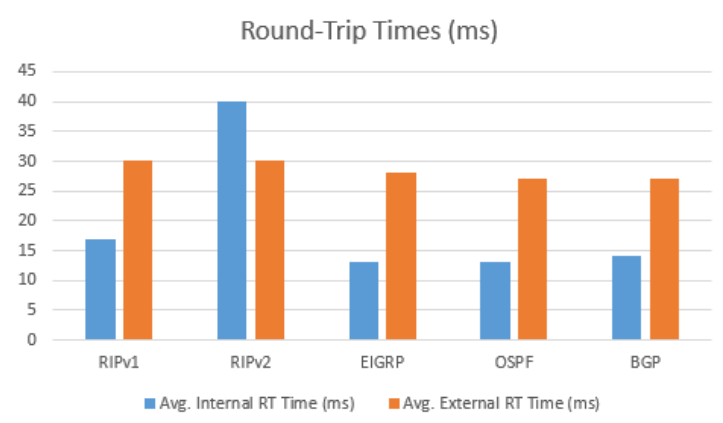

Fig. 10. Average round-trip times graphic of dynamic routing protocols on star topology.

As given in Fig. 10, in star topology, OSPF and EIGRP again have the lowest Average Internal Round-Trip Time values between interior gateway protocols like in ring topology. Consequently routers that run OSPF and EIGRP protocols, have the shortest package transfer time or in other words have the fastest package transfer value. BGP, which is an exterior gateway protocol, has a short Average Internal Round-Trip Time. This leads a fast package transfer between routers that run BGP protocol, which is designed to work between autonomous systems. In this way, network connection's continuity and speed will be provided. Again as shown in Fig. 10, like in ring topology, Average External Round-Trip Time has minimum values in star topology with routers which uses OSPF and EIGRP protocols. Consequently routers which use OSPF and EIGRP, transfers packages to remote networks considerably slow when compared to routers which use other interior gateway protocols. The reason of this is, EIGRP and OSPF is more 
efficient when compared to RIP protocols [7]. BGP, which is an exterior protocol, has low value of Average External Round-Trip Time. Therefore, packages transferred between autonomous systems and remote networks would be fast and this leads to a fast connection.

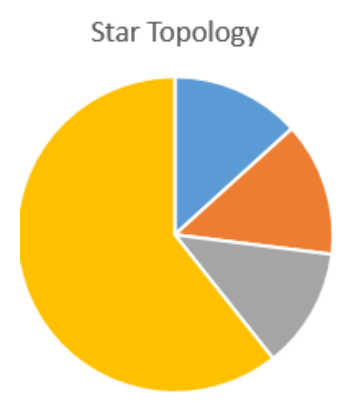

" RIPV1 $=$ RIPV2 = EIGRP $=$ OSPF

Fig. 11. Analysis of interior gateway protocols on star topology.

As presented in Fig. 11, considering the Performance Analysis of Interior Gateway Protocols on Star Topology values, one can clearly say that, the most efficient protocol between Interior Gateway Dynamic Routing Protocols in star topology is OSPF. Therefore OSPF can not only chosen in ring topology, but also in star topology.

\section{Analysis of Dynamic Routing Protocols on Mesh Topology}

The results of analysis has obtained by using the same mesh topology in every experiment but different dynamic routing protocols in each time. During the analysis, the configurations of RIPv1, RIPv2, EIGRP, OSPF and BGP was performed and they were compared with each other.

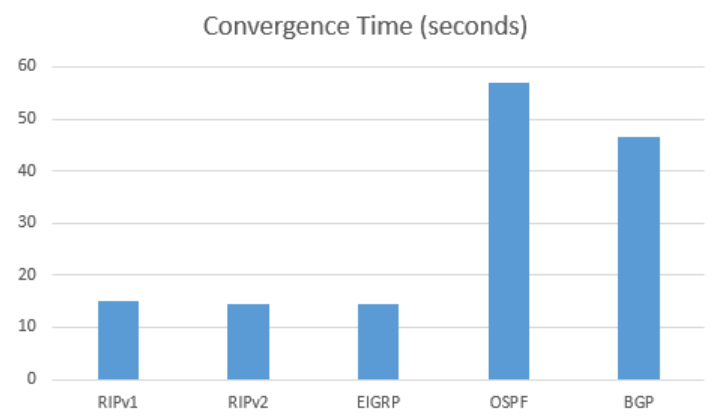

Fig. 12. Convergence time graphic of dynamic routing protocols on mesh topology.

As illustrated in Fig. 12, EIGRP has the lowest convergence time value between interior gateway protocols in mesh topology, like it was in ring and star topologies. RIP protocols also have approximately the same value in terms of convergence time. In mesh topology, routers which run EIGRP, RIPv1 and RIPv2 protocols can create and update their routing tables faster than routers which run OSPF protocol. The reason of this quick discovery of network in mesh topology is that, these protocols are distance vector protocols. In a mesh topology, every node is connected one by one with each other. Distance vector protocols periodically updates every change in the network, therefore it takes considerably short time to learn a change in the network. BGP, which is an exterior gateway protocol, has a high convergence value, because it runs a complex algorithm to learn networks.

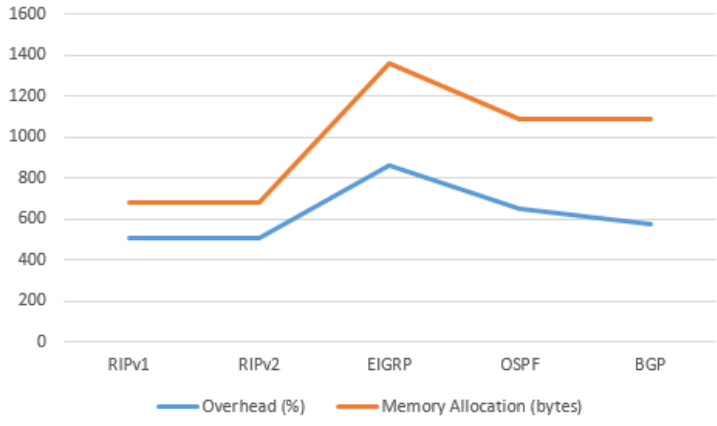

Fig. 13. Overhead and memory allocation graphic of dynamic routing protocols on mesh topology.

As shown in Fig. 13, considering the overhead, EIGRP has the highest overhead value between interior gateway protocols [8]. Same events about overhead examinations, mentioned in other topologies are valid in Mesh topology also. Being a distance vector, increases overhead of EIGRP protocol. BGP, which is an exterior gateway protocol, again has a low overhead value. The aim of this low overhead value is that to provide a fast and high quality internet connection. Again as shown in Fig. 13, considering the memory usage, EIGRP has the highest value of memory use between interior gateway protocols in mesh topology also, like was in other topologies [9]. The reason of this high value is that EIGRP periodically gets updates from neighbour routers. BGP, which is an exterior gateway protocol, has a medium value of memory usage. This arises from the architecture (every node is connected to each other one by one) of mesh topology. Learning and updating every node requires more memory usage so this leads an increase in memory usage in mesh topology in BGP protocol when compared to other topology behaviours of BGP.

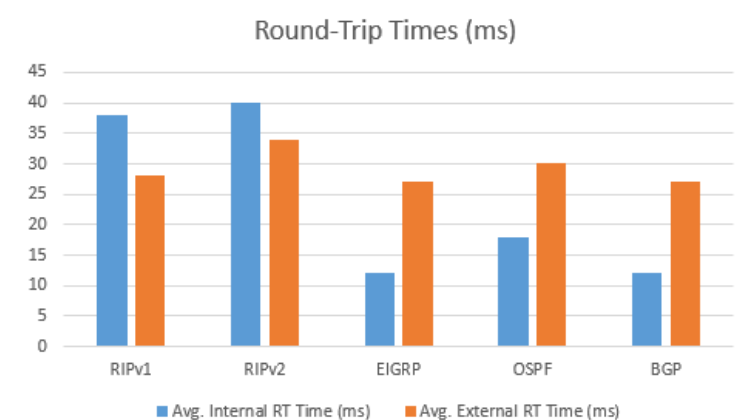

Fig. 14. Average round-trip times graphic of dynamic routing protocols on mesh topology.

As shown in Fig. 14, regarding the Average Internal Round-Trip Time, OSPF and EIGRP has the minimum values between interior gateway protocols in mesh topology also, like it was in other topologies. Consequently routers that run OSPF and EIGRP protocols, have shorter package transfer time or in other words have faster package transfer value. BGP, which is an exterior gateway protocol, has a short Average Internal Round-Trip Time. Routers that runs BGP protocols has maximum package transfer speed value in mesh topology. The reason of this speed is that, BGP is designed to work efficiently and effectively in complex topology networks. Again as shown in Fig. 14, considering the Average External Round-Trip Time in mesh topology, RIPv1 and EIGRP protocols have the lowest value between interior 
gateway protocols. Consequently, transfer speed of routers running these protocols are high while transferring to distant networks. On the other hand, RIPv1 protocol will be inadequate in more complex topologies, so in this cases EIGRP must be used. BGP, which is an external gateway protocol, has the lowest Average Internal Round-Trip Time value. This leads to a very fast data communication between BGP running routers in complex networks.

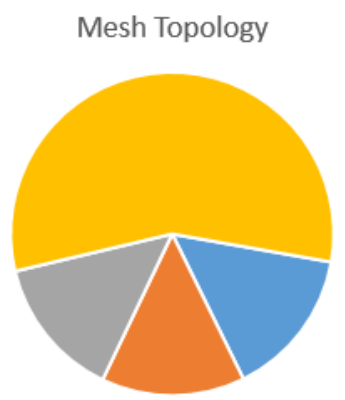

" RIPV1 " RIPv2 | EIGRP \#OSPF

Fig. 15. Analysis of interior gateway protocols on mesh topology.

As presented in Fig. 15, considering the values in pie chart, OSPF is again the most efficient protocol between interior Gateway Dynamic Routing Protocols in mesh topology [9]. OSPF can be chosen for running routing protocol in mesh topology also, like was in the other topologies.

\section{CONCLUSION}

The analysis was performed with using 3 types of topologies which are ring, star and mesh. Each protocol was applied on each topology separately, and results were created with using the obtained results.

Four interior gateway protocols which are RIPv1, RIPv2, EIGRP and OSPF, and one exterior gateway protocol which is BGP was used during the analysis.

As shown in Fig. 7, Fig. 11 and Fig. 15, when all of the interior gateway protocols were examined on 3 types of topologies, we can say that OSPF has the highest performance among all of the interior gateway protocols. Therefore, using OSPF on interior networks is the best choice.

BGP was created as a reason of the performances of the interior gateway protocols, which are RIPv1, RIPv2, EIGRP and OSPF, is not enough. BGP is used between Autonomous Systems (ASs), the fundamental networks which are components of the internet architecture. Therefore, BGP is an exterior gateway protocol and its performance is higher than all of the interior gateway protocols. It is shown that, BGP performs with the highest performance.

We believe that the analysis performed here can be useful for network designers, administrators when considering various topologies and routing protocols for their specific needs.

\section{REFERENCES}

[1] U. D. Black, IP Routing Protocols, 2000.

[2] B. Albrightson et al., "EIGRP-A fast routing protocol based on distance vectors," University of California Santa Cruz, 1994.

[3] J. T. Moy, "OSPF: Anatomy of an internet routing protocol," 1998.

[4] S. G. Thorenoor, "Dynamic routing protocol implementation decision between EIGRP, OSPF and RIP based on technical background using OPNET modeler," Wipro Technol., Bangalore, India, April 2010.

[5] M. N. Islam and A. U. Ashique, "Simulation based EIGRP over OSPF performance analysis," May 2014.

[6] J. Baranski, M. Crocker, and G. Lazarou, "Dynamic routing protocol performance in a fault-tolerant Ethernet-based IP network," Department of Electrical and Computer Engineering, Mississippi State University, Mississippi State.

[7] C. Wijaya, "Performance analysis of dynamic routing protocol EIGRP and OSPF in IPv4 and IPv6 network," Parahyangan Catholic Univ., Bandung, Indonesia, December 2011.

[8] B. Bostanci and E. Bostanci, "An evaluation of classification algorithms using Mc Nemar's test".

[9] S. Sendra, P. A. Fernandez, M. A. Quilez, and J. Lloret, "Study and performance of interior gateway IP routing protocols," Integrated Management Coastal Research Institute, Polytechnic University of Valencia, Gandia, Valencia, 2010.

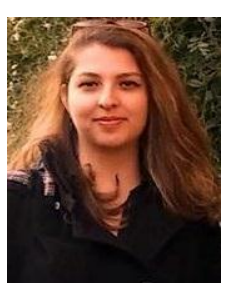

Irem Dogan is studying in Computer Engineering Department of Ankara University, Turkey. She is a final year undergraduate student.

At the same time, she is currently working at Cisco Systems as the lab administrator and technical intern. She completed her first internship at IT Department of Istanbul Technical University as the network engineer, Turkey in 2014. She researched for TRILL (Transparent Interconnection of Lots of Links) on different network topologies and she documented this research for IT usage of Istanbul Technical University.

She completed her second internship at IT Department of ICterra Software as a system engineer, Turkey in 2015. She was assigned to a project which was about system and network security and she studied with some honeypots for detecting various attacks.

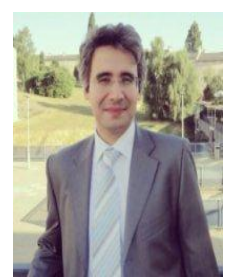

Erkan Bostanci received a BSc degree in Computer Engineering Department from Ankara University, Turkey in 2007. Consequently, he joined the same department as a Research Assistant and completed his MSc degree on real-time battlefield simulation in 2009. He obtained his PhD degree from the School of Computer Science and Electronic Engineering, University of Essex, United Kingdom in 2014 with his thesis on real-time user tracking for augmented reality.

He started working with the Gendarmarie Schools Command as a Planning Officer Designate in June, 2014 where he conducted the research for developing a vision-based system for analysing crime scenes. He has been promoted to Second Lieutenant in January, 2015. Having completed his military service, he currently continues his post in Ankara University as an Assistant Professor.

His research interests include different yet closely related aspects of computer science from image processing, computer vision and graphics to artificial intelligence and fuzzy logic as well as mathematical modelling and statistical analysis. He recently developed a vision-based user tracking system for various augmented reality applications for cultural heritage in particular. He setup the SAAT laboratory in the department for conducting research with the main aim for incorporating AI approaches for solving a wide range of real world problems.

Dr. Bostanci has been involved in technical committees for several conferences and acted as a reviewer for various journals. 